Pacific Journal of Mathematics

ON THE GENUS OF THE COMPOSITION OF TWO GRAPHS 


\title{
ON THE GENUS OF THE COMPOSITION OF TWO GRAPHS
}

\author{
ARthur T. White
}

\begin{abstract}
Given two graphs $G$ and $H$, a new graph $G(H)$, called the composition (or lexicographic product) of $G$ and $H$, can be formed. In this paper, a formula is developed to give the genus for a large class of lexicographic products. In the simplest special case, the genus of the product is given by the first Betti number of one of the factors.
\end{abstract}

In the present context, a graph is a finite 0- or 1- complex. For terms not defined below, see [2] and [6].

The genus, $\gamma(G)$, of a graph $G$ is the minimum genus among the genera of all closed orientable 2-manifolds $M$ in which $G$ can be imbedded. An imbedding of $G$ in $M$ is said to be minimal if $M$ has genus $\gamma(G)$. The first Betti number, $\beta(G)$, of a graph $G$ is given by $\beta(G)=q-p+k$, where $G$ has $q$ edges, $p$ vertices, and $k$ components; $\beta(G)$ counts the number of independent cycles in $G$. Given two graphs $G$ and $H$ with disjoint vertex sets $V(G), V(H)$ and edge sets $E(G)$, $E(H)$ respectively, the composition (or lexicographic product) $G(H)$ has vertex set given by the cartesian product $V(G) \times V(H)$, with two vertices $\left(u_{i}, v_{j}\right)$ and $\left(u_{k}, v_{m}\right)$ adjacent in $G(H)$ if and only if either: (i) $u_{i}=u_{k}$ and $v_{j} v_{m}$ is in $E(H)$, or (ii) $u_{i} u_{k}$ is in $E(G)$. For example, the regular complete $m$-partite graph on $m n$ vertices is just the composition $K_{m}\left(\bar{K}_{n}\right)$, where $K_{s}$ denotes the complete graph on $s$ vertices, and $\overline{K_{s}}$ denotes the complement of $K_{s}$ (a 0 -complex).

We will also employ the following notions. If $G$ is imbedded in $M$, the components of $M-G$ are called regions. A region bounded by a circuit of length $3(4)$ in $G$ is said to be triangular (quadrilateral). The number of triangular (quadrilateral) regions in a given imbedding is denoted by $r_{3}\left(r_{4}\right)$. In general, $r_{k}$ designates the number of regions having a connected boundary consisting of $k$ edges of $G$, and $r$ denotes the total number of regions. It is well known (see, for example, [6]) that, for a minimal imbedding of a connected graph $G$ having $p$ vertices and $q$ edges, the Euler formula $p-q+r=2-2 \gamma(G)$ applies. Also, it is easy to show that $2 q=\sum_{i \geqq 3} i r_{i}$. We note that a 3-cycle in a graph $G$ need not bound a triangular region in a given minimal imbedding of $G$. For example, there are 35 3-cycles in $K_{7}$; yet any minimal imbedding of $K_{7}$ has $r=r_{3}=14$.

The following result of Battle, Harary, Kodama, and Youngs [1] will be useful: 
THEOREM. The genus of a graph is the sum of the genera of its components.

We are now prepared to state the main result.

TheOREM. Let $G$ have $p$ vertices of positive degree, $q$ edges, $k$ nontrivial components, and no 3-cycles. Let $H$ have $2 n(n \geqq 1)$ vertices and maximum degree less than two. Then $\gamma(G(H))=k+n(n q-p)$.

Proof. Let $G$ have nontrivial components $C_{i}, i=1, \cdots, k$; then $G(H)$ has nontrivial components $C_{i}(H), i=1, \cdots, k$. It will suffice to prove the theorem for $G$ connected, since then (by the result of Battle, Harary, Kodama and Youngs):

$$
\begin{aligned}
\gamma(G(H)) & =\sum_{i=1}^{k} \gamma\left(C_{i}(H)\right) \\
& =\sum_{i=1}^{k}\left(1+n\left(n q_{i}-p_{i}\right)\right) \\
& =k+n(n q-p) .
\end{aligned}
$$

We therefore assume $G$ to be connected. Let $V(G)=\left\{u_{1}, \cdots, u_{p}\right\}$, and $V(H)=\left\{v_{1}, \cdots, v_{2 n}\right\}$.

Suppose the vertices $\left(u_{i}, v_{j}\right),\left(u_{k}, v_{m}\right)$, and $\left(u_{r}, v_{s}\right)$ form a 3-cycle in $G(H)$. Since there are no 3-cycles in $G$, the vertices $u_{i}, u_{k}$, and $u_{r}$ cannot be distinct in $V(G)$. Hence every 3-cycle in $G(H)$ must contain an edge of the form $\left(u_{i}, v_{j}\right)\left(u_{i}, v_{m}\right)$. There are exactly pe such edges in $G(H)$, where $e$ designates the number of edges in $H(0 \leqq e \leqq n)$. Since each one of these edges can appear in the boundary of at most 2 triangular regions, it follows that $r_{3}{ }^{*} \leqq 2 p e$ in any imbedding of $G^{*}=G(H)$. (A parameter with (without) an asterisk will apply to graph $\left.G^{*}(G)\right)$.

We will construct an imbedding of $G^{*}$ so that $r_{3}^{*}=2 p e$ and $r_{4}^{*}=$ $r^{*}-2 p e$; since $r^{*}=\sum_{i \geq 3} r_{i}^{*}$ and $2 q^{*}=\sum_{i \geqq 3} i r_{i}^{*}, r^{*}$ will be maximal for such an imbedding. Then, by the Euler formula, the imbedding itself will be minimal. Now, for $G^{*}, p^{*}=2 n p$, and $q^{*}=p e+4 n^{2} q$. Also, if $r_{3}^{*}=2 p e=r^{*}-r_{4}^{*}$, then $r^{*}=p e+2 n^{2} q$, since $2 q^{*}=2 p e+$ $8 n^{2} q=3(2 p e)+4\left(r^{*}-2 p e\right)$. Then, from the Euler formula,

$$
\begin{aligned}
\gamma\left(G^{*}\right) & =1+1 / 2\left(q^{*}-p^{*}-r^{*}\right) \\
& =1+1 / 2\left(p e+4 n^{2} q-2 n p-\left(p e+2 n^{2} q\right)\right) \\
& =1+n(n q-p)
\end{aligned}
$$

We now construct such an imbedding. Let the edges of $G$ be designated by $x_{1}, \cdots, x_{q}$. For each edge there is a subgraph of $G(H)$ isomorphic to the complete bipartite graph $K_{2 n 2 n}$. Imbed $q$ copies of $K_{2 n, 2 n}$, minimally, in $q$ closed orientable 2 -manifolds $M_{1}, \cdots, M_{q}$ of 
genus $(n-1)^{2}$ each, in the fashion described by Ringel [3]. Select these 2-manifolds so that each is exterior to any other. Each imbedding has $r^{\prime}=r_{4}^{\prime}=2 n^{2}$, and it has been shown in [5] that the $2 n^{2}$ quadrilateral regions can be partitioned into $2 n$ mutually disjoint sets of $n$ regions each, each set containing all $4 n$ vertices of the graph $K_{2 n, 2 n}$. Furthermore, in any region, diagonally opposite vertices are in the same part of the vertex set partition for $K_{2 n, 2 n}$.

Suppose edges $x_{i}$ and $x_{j}$ are adjacent in $G$. We make $2 n$ vertex identifications between $M_{i}$ and $M_{j}$ as follows. Select one set of $n$ quadrilateral regions in $M_{i}$ and the $2 n$ vertices of one part of the vertex set partition for $K_{2 n, 2 n}$ from the boundaries of these regions (two diagonally opposite vertices are selected from the boundary of each region). Make similar selections in $M_{j}$. Now attach $n$ tubes between $M_{i}$ and $M_{j}$, one tube for each pair of regions (one from each 2-manifold) that we have selected. The first such tube may be attached as follows. Let region $R^{i}$ in $M_{i}$ correspond to region $R^{j}$ in $M_{j}$. Let $C^{h}$ be a simple closed curve bounding the open disk $D^{h}$ interior to $R^{h}, h=i, j$. Let $T$ be a topological cylinder, with bases $C_{i}$ and $C_{j}$, such that $\left(M_{i} \cup M_{j}\right) \cap T=C_{i} \cup C_{j}$. Form $\left(M_{i}-D^{i}\right) \cup$ $\left(M_{j}-D^{j}\right) \cup T$. It is clear how to add the remaining tubes. The result is a closed orientable 2 -manifold $M$ (of genus $2(n-1)^{2}+n-1$ ).

We now make two vertex identifications per tube, as indicated by the sequence of operations in Figure 1.

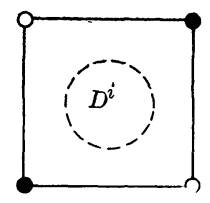

1a

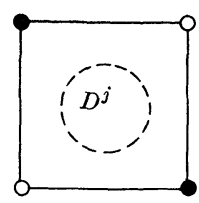

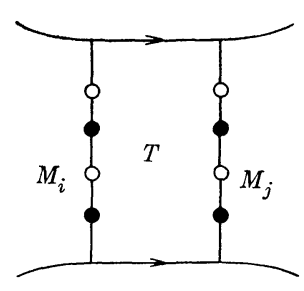

$1 \mathrm{~b}$

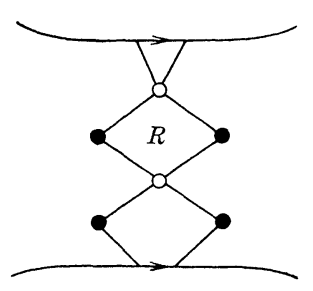

$1 \mathrm{c}$

FIGURE 1

This process destroys two quadrilaterals and creates two new quadrilaterals for each tube. Furthermore, the two identifications for each tube yield two vertices diagonally opposite in a common region $R$. If edge $x_{k}$ is also adjacent to $x_{i}$ (and to $x_{j}$ ) in $G$, there are now $n$ regions available on the 2-manifold $M$ with which to make the appropriate $2 n$ identifications with the 2-manifold $M_{k}$. From these $n$ regions on $M$, we select the diagonally opposite vertices that resulted from the first identification. It is clear that this process may be continued until a quadrilateral imbedding of $G\left(\bar{K}_{2 n}\right)$ results. We need only insure that, for 2-manifold $M_{i}$ corresponding to edge $x_{i}=\left[u_{i 1}, u_{i 2}\right]$ in $G$, if we selected the $2 n$ vertices of one part of the vertex-set 
partition of $K_{2 n, 2 n}$ with which to make the identifications at $u_{i 1}$ in $G$, then we must select the $2 n$ vertices of the second part of the vertex-set partition of $K_{2 n, 2 n}$ with which to make the identifications at $u_{i 2}$ in $G$.

Corresponding to each vertex of $G$, there is now a copy of $\bar{K}_{2 n}$, within which the $e$ edges of $H$ may be added. Each such edge converts one quadrilateral region of the imbedding of $G\left(\bar{K}_{2 n}\right)$ into two triangular regions. The result is an imbedding of $G^{*}$ having $r_{3}^{*}=2 p e$ and $r_{4}^{*}=r^{*}-2 p e$, as desired. This completes the proof.

We note that the value $r^{*}=2 q n^{2}+p e$ may be verified by a direct count, since $r_{4}^{*}=q\left(2 n^{2}\right)$ - pe. Also, the genus of $G(H)$ may be computed directly, for this construction, without recourse to any Euler type formula. The contributions to the genus are of three types:

(i) $q(n-1)^{2}$, representing the collective genera of the $q 2$ manifolds with which we began our construction;

(ii) $(2 q-p)(n-1)$, representing the contribution of the $2 q-p$ sets of $2 n$ vertex identifications each, each "bundle" of $n$ tubes adding $n-1$ to the genus;

(iii) $\beta(G)=q-p+1$, representing the contribution of the bundles of tubes taken collectively.

Adding, we find:

$$
\begin{aligned}
\gamma(G(H)) & =q(n-1)^{2}+(2 q-p)(n-1)+(q-p+1) \\
& =1+n(n q-p)
\end{aligned}
$$

It is no surprise that the formula $\gamma\left(K_{2 n, 2 n}\right)=(n-1)^{2}$ is included in the above theorem. For the case where $G$ is the cycle $C_{s}$ and $H=\bar{K}_{2 n}$, we may combine the theorem with the result of Ringel and Youngs [4] that $\gamma\left(K_{3}\left(\overline{K_{m}}\right)\right)=((m-1)(m-2)) / 2$ to establish the following:

COROLLARY 1.

$$
\gamma\left(C_{s}\left(\bar{K}_{2 n}\right)\right)=\left\{\begin{array}{l}
1+n(2 n-3), \text { if } s=3 \\
1+n s(n-1), \text { if } s \geqq 4 .
\end{array}\right.
$$

In the situation where $G$ is the complete bipartite graph $K_{r, s}$ and $H$ is as in the statement of the theorem, we have:

CoROLlary 2. $\gamma\left(K_{r, s}(H)\right)=(n r-1)(n s-1)$.

We list here only one other result, for the special case $n=1$ of the theorem:

Corollary 3. Let $G$ be a graph containing no 3-cycles. Then $\gamma\left(G\left(K_{2}\right)\right)=\gamma\left(G\left(\overline{\left.K_{2}\right)}\right)=\beta(G)\right.$. 


\section{REFERENCES}

1. J. Battle, F. Harary, Y. Kodama, and J. W. T. Youngs, Additivity of the genus of a graph, Bull. Amer. Math. Soc., 68 (1962), 565-568.

2. F. Harary, Graph Theory, Addison-Wesley, Reading (1969).

3. G. Ringel, Das Geschlecht des Vollständigen Paaren Graphen, Abh. Math. Sem. Univ. Hamburg, 28 (1965), 139-150.

4. G. Ringel and J. W. T. Youngs, Das Geschlecht des Symmetrische Vollständige DreiFarbaren Graphen. Comment. Math. Helv., 45 (1970), 152-158.

5. A. T. White, The genus of repeated cartesian products of bipartite graphs. Trans. Amer. Math. Soc., 151 (1970), 393-404.

6. J. W. T. Youngs, Minimal imbeddings and the genus of a graph, J. Math. Mech., 12 (1963), 303-315.

Received May 22, 1970

WESTERN MICHIGAN UNIVERSITY 



\section{PACIFIC JOURNAL OF MATHEMATICS}

\section{EDITORS}

H. SAMELSON

Stanford University

Stanford, California 94305

C. R. Hовву

University of Washington

Seattle, Washington 98105
J. DugundJI

Department of Mathematics

University of Southern California

Los Angeles, California 90007

RICHARD ARENS

University of California

Los Angeles, California 90024

\section{ASSOCIATE EDITORS}
E. F. BECKENBACH
B. H. NeumanN
F. WOLF
K. YOSHIDA

\section{SUPPORTING INSTITUTIONS}

UNIVERSITY OF BRITISH COLUMBIA

CALIFORNIA INSTITUTE OF TECHNOLOGY

UNIVERSITY OF CALIFORNIA

MONTANA STATE UNIVERSITY

UNIVERSITY OF NEVADA

NEW MEXICO STATE UNIVERSITY

OREGON STATE UNIVERSITY

UNIVERSITY OF OREGON

OSAKA UNIVERSITY
UNIVERSITY OF SOUTHERN CALIFORNIA

STANFORD UNIVERSITY

UNIVERSITY OF TOKYO

UNIVERSITY OF UTAH

WASHINGTON STATE UNIVERSITY

UNIVERSITY OF WASHINGTON

$\stackrel{*}{*} \stackrel{*}{*} \stackrel{*}{*}{ }^{*}$ AMERICAN MATHEMATICAL SOCIETY

NAVAL WEAPONS CENTER 


\section{Pacific Journal of Mathematics}

\section{Vol. 41, No. $1 \quad$ November, 1972}

Anatole Beck and Peter Warren, Weak orthogonality.................

Jonnie Bee Bednar and Howard E. Lacey, Concerning Banach spaces whose duals are abstract $L$-spaces.............................

Louis Harvey Blake, Canonical extensions of measures and the extension of regularity of conditional probabilities..........................

R. A. Brooks, Conditional expectations associated with stochastic processes ..........................................

Theodore Allen Burton and Ronald Calvin Grimmer, On the asymptotic behavior of solutions of $x^{\prime \prime}+a(t) f(x)=e(t) \ldots \ldots \ldots \ldots \ldots \ldots$

Stephen LaVern Campbell, Operator-valued inner functions analytic on the closed disc ............................................

Yuen-Kwok Chan, A constructive study of measure theory...

Alexander Munro Davie and Bernt Karsten Oksendal, Peak interpolation sets for some algebras of analytic functions ...................

H. P. Dikshit, Absolute total-effective $\left(N, p_{n}\right)(c, 1)$ method ...............

Robert E. Edwards, Edwin Hewitt and Kenneth Allen Ross, Lacunarity for

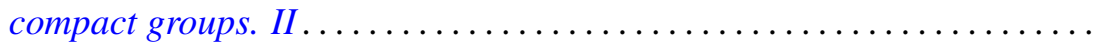

James Daniel Halpern, On a question of Tarski and a maximal theorem of Kurepa

Gerald L. Itzkowitz, A characterization of a class of uniform spaces that admit an invariant integral

Mo Tak Kiang, Semigroups with diminishing orbital diameters ..

Glenn Richard Luecke, A class of operators on Hilbert space ...

R. James Milgram, Group representations and the Adams spectral sequence. . .

G. S. Monk, On the endomorphism ring of an abelian p-group, and of a large subgroup...

Yasutoshi Nomura, Homology of a group extension ...

R. Michael Range, Approximation to bounded holomorphic functions on strictly pseudoconvex domains...

Norman R. Reilly, Inverse semigroups of partial transformations and $\theta$-classes.

Chris Rorres, Strong concentration of the spectra of self-adjoint operators

Saharon Shelah, A combinatorial problem; stability and order for models and theories in infinitary languages.

George Gustave Weill, Vector space decompositions and the abstract

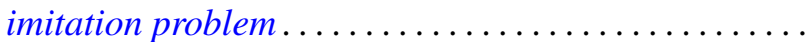

